\title{
BACKWARD STOCHASTIC DIFFERENCE EQUATIONS FOR DYNAMIC CONVEX RISK MEASURES ON A BINOMIAL TREE
}

\author{
ROBERT J. ELLIOTT, ${ }^{*}$ University of Adelaide and University of Calgary \\ TAK KUEN SIU,** Macquarie University and City University London \\ SAMUEL N. COHEN, ${ }^{* * *}$ University of Oxford
}

\begin{abstract}
Using backward stochastic difference equations (BSDEs), this paper studies dynamic convex risk measures for risky positions in a simple discrete-time, binomial tree model. A relationship between BSDEs and dynamic convex risk measures is developed using nonlinear expectations. The time consistency of dynamic convex risk measures is discussed in the binomial tree framework. A relationship between prices and risks is also established. Two particular cases of dynamic convex risk measures, namely risk measures with stochastic distortions and entropic risk measures, and their mathematical properties are discussed.
\end{abstract}

Keywords: Dynamic convex risk measure; conditional nonlinear expectation; binomial tree; backward stochastic difference equation; stochastic distortion probability

2010 Mathematics Subject Classification: Primary 91G20

Secondary $91 \mathrm{G} 80 ; 60 \mathrm{H} 07$

\section{Introduction}

Risk management is an important issue in the banking, finance, and insurance industries. Recent financial crises, collapses of major financial institutions and insurance companies, such as the global financial crisis of 2008, the Asian financial crisis, collapses of Long-Term Capital Management (LTCM), AIG and Lehman Brothers, the turmoil at Barings and Orange County, may be partly attributed to inappropriate risk measurement. International regulatory bodies, such as the Bank of International Settlements (BIS) and the Basel Committee for Banking Supervision, have produced reports with detailed guidelines on quantitative methods, techniques, and practices for risk management. Among various issues of risk management, developing appropriate quantitative models for risk measurement is of importance. Due to the proliferation of financial and insurance products, risk measurement issues have become more sophisticated.

Value at Risk (VaR) has emerged as a popular tool for risk measurement among market practitioners in the banking, finance, and insurance industries. Its origin may be traced back to the famous 4:15 p.m. report at JP Morgan, where an early application of VaR was presented.

\footnotetext{
Received 1 November 2013; revision received 6 August 2014.

* Postal address: School of Mathematical Sciences, University of Adelaide, SA 5005, Australia.

Email address: relliott@ucalgary.ca

** Postal address: Department of Applied Finance and Actuarial Studies, Faculty of Business and Economics, Macquarie University, Sydney, NSW 2109, Australia.

*** Postal address: Mathematical Institute, University of Oxford, Andrew Wiles Building, Woodstock Road, Oxford OX2 6GG, UK.
} 
Although VaR is a popular tool for risk measurement in practice, it has been noted that VaR has some defects. Artzner et al. [1] introduced the class of coherent risk measures, which satisfy the properties of subadditivity, translation invariance, positive homogeneity, and monotonicity and obtained a representation for coherent risk measures. Subadditivity is one of the four desirable properties. Intuitively, it states that the diversification between two risky positions should always reduce risk. Artzner et al. [1] pointed out that VaR does not, in general, satisfy the subadditivity property, especially when the portfolio contains derivative instruments. Föllmer and Schied [23] and Frittelli and Rosazza-Gianin [25] argued that in many cases the risk of a trading portfolio might increase in a nonlinear fashion with the size of the portfolio due to the additional liquidity risk of a large portfolio. They relaxed the subadditive and positive homogeneous properties and replaced them with the convexity property. The notion of convex risk measures, which extends the concept of coherent risk measures, was then introduced. Föllmer and Schied [23] and Frittelli and Rosazza-Gianin [25] established independently a representation for convex risk measures based on probability measures on the underlying space of 'scenarios' and a penalty function. Interested readers may refer to Elliott and Kopp [18] for a discussion of coherent risk measures and to Föllmer and Schied [24] for an exposition of convex risk measures.

Both coherent and convex risk measures were initially introduced in a static setting. However, in practice, risk measurement should be performed dynamically over time. Consequently, there is a need for dynamic risk measures. Some versions of dynamic coherent risk measures appeared in [2], [5], [12], and [34]-[36]. Frittelli and Rosazza-Gianin [26], Detlefsen and Scandolo [15], Klöppel and Schweizer [28], Jobert and Rogers [27], and others studied dynamic convex risk measures from a theoretical perspective; see also [19], [20], and [33]. Time-consistency is an important property of dynamic risk measures. This property has been defined in a number of works including, e.g. [2], [11], [26], [32], and [39]. These works also defined dynamic risk measures and studied their time consistency. It was noted in Rosazza-Gianin [32] that the notion of time consistency is related to the 'filtration consistency' introduced by Coquet $e t$ al. [11] and the recursivity of Artzner et al. [2]. Artzner et al. [2] and Delbaen [13] stressed that time-consistency is not satisfied by all the dynamic risk measures. It is satisfied only by those dynamic risk measures with a set of probability measures or generalized scenarios satisfying the ' $m$-stability' property, which, roughly speaking, requires that the generalized scenario obtained by 'pasting ad hoc' two probability measures in the set of generalized scenarios again falls into the same set. The ' $m$-stability' and pasting of probability measures were discussed in Delbaen [13]. Rosazza-Gianin [32] established the link between dynamic convex/coherent risk measures and conditional $g$-expectations in a continuous-time Brownian filtration setting. The conditional $g$-expectations satisfy the backward stochastic differential equations driven by a standard Brownian motion under certain conditions. For an excellent exposition on the use of backward stochastic differential equations in finance, we refer the reader to [17].

In this paper, we adopt backward stochastic difference equations (BSDEs) to study dynamic convex risk measures in a simple discrete-time, binomial tree model. Dynamic convex risk measures are linked to solutions of BSDEs via nonlinear expectations in the binomial tree model. The necessary and sufficient conditions for the time consistency of dynamic convex risk measures are given. We also discuss the relationship between prices and risks, and derive a decomposition for the difference between a price and a risk into different risk-premium components in the binomial tree framework. Two particular cases of dynamic convex risk measures are considered. The first one is a risk measure introduced by stochastic distortion probabilities and the second one is an entropic risk measure. Time consistency of these two 
risk measures is discussed. The one-step law invariance of dynamic convex risk measures is also analysed.

The rest of the paper is structured as follows. In the next section we present the BSDEs under the binomial tree model. In Section 3 we discuss the link between the BSDEs and dynamic convex risk measures. In Section 4 we discuss the relationship between prices and risks. The two particular cases and some mathematical properties of risk measures are considered in Section 5 .

\section{BSDEs in a binomial tree}

In this section we present a discrete-time, binomial model and some basic results for BSDEs in the binomial model. These results include the existence and uniqueness of the solutions of the BSDEs as well as a comparison theorem.

As usual, let $(\Omega, \mathcal{F}, \mathbb{P})$ be a probability space describing uncertainty in the binomial model, where $\mathbb{P}$ is a real-world probability measure. Denote the time index set $\{0,1,2, \ldots, T\}$ by $\mathcal{T}$. Consider a Bernoulli process $\mathcal{X}:=\left\{\mathcal{X}_{t} \mid t \in \mathcal{T} \backslash\{0\}\right\}$ on $(\Omega, \mathcal{F}, \mathbb{P})$ with state space $\{0,1\}$. Let $\mathbb{F}:=\left\{\mathcal{F}_{t} \mid t \in \mathcal{T}\right\}$ be the natural filtration generated by the process $\mathcal{X}$. To simplify the discussion, take $\mathcal{F}=\mathscr{F}_{T}$ and assume that $\mathcal{F}_{0}$ is trivial. This defines a martingale difference process, denoted by $M:=\left\{M_{t} \mid t \in \mathcal{T} \backslash\{0\}\right\}$, as

$$
M_{t}=\mathcal{X}_{t}-\mathbb{E}\left[\mathcal{X}_{t} \mid \mathcal{F}_{t-1}\right] .
$$

Write $p_{t-1}=\mathbb{E}\left[\mathcal{X}_{t} \mid \mathcal{F}_{t-1}\right]=\mathbb{P}\left(\mathcal{X}_{t}=1 \mid \mathcal{F}_{t-1}\right)$ so that $p_{t-1}$ is $\mathcal{F}_{t-1}$ measurable, and $M_{t}=1-p_{t-1}$ when $\mathcal{X}_{t}=1$. The process $M$ is used as the basic martingale difference process of the BSDE to be defined later in this section.

We shall henceforth assume that, for the measures considered, $p_{t} \in(0,1)$ for all $t$ and $\omega$. This assumption ensures that each path of our binomial tree is associated with a positive probability, and ensures that all probability measures on our sample space $(\Omega, \mathcal{F})$ are absolutely continuous with respect to $\mathbb{P}$. Consequently, our results will hold for all $\omega$, rather than for almost all $\omega$.

The following construction is standard.

Lemma 1. For any $\mathbb{F}$-adapted stochastic process $\tilde{p}:=\left\{\tilde{p}_{t} \mid t \in \mathcal{T} \backslash\{T\}\right\}$ on $(\Omega, \mathcal{F}, \mathbb{P})$ with state space $[0,1]$, there is a probability measure $\tilde{\mathbb{P}}$ on $(\Omega, \mathcal{F})$ such that

$$
\tilde{\mathbb{E}}\left[X_{t+1} \mid \mathscr{F}_{t}\right]=\tilde{p}_{t}
$$

Here $\tilde{\mathbb{E}}$ is the expectation under $\tilde{\mathbb{P}}$.

Proof. Define

$$
\left.\frac{\mathrm{d} \tilde{\mathbb{P}}}{\mathrm{d} \mathbb{P}}\right|_{\mathcal{F}_{T}}:=\prod_{i=1}^{T-1}\left(\frac{\tilde{p}_{i}}{p_{i}}\right)^{\chi_{i+1}}\left(\frac{1-\tilde{p}_{i}}{1-p_{i}}\right)^{1-\chi_{i+1}} .
$$

In the sequel, some basic results for existence and uniqueness of solutions to BSDEs are given in the binomial context. Conditions under which a comparison theorem will hold are provided. The theory of discrete-time BSDEs and its relationships with dynamic risk measures were discussed in Cohen and Elliott [8], [10], and Cheridito and Stadje [6].

We begin by defining the following version of the Malliavin difference, which will be used in the martingale representation theorem for Markov chains. A full treatment of the discrete Malliavin calculus (scaled to fit an alternate notation) can be found in Privault [31, Chapter 1]. 
Definition 1. Let $Q$ be a $\mathcal{F}_{T}$-measurable random variable. By the Doob-Dynkin lemma, it has a representation $Q=f\left(\mathcal{X}_{1}, \mathcal{X}_{2}, \ldots, \mathcal{X}_{T}\right)$. Define the up- and down-values $Q^{t+}$ and $Q^{t-}$ by the random variables

$$
\begin{aligned}
Q^{t+} & :=f\left(X_{1}, \ldots, X_{t-1}, 1, \chi_{t+1}, \ldots, X_{T}\right), \\
Q^{t-} & :=f\left(X_{1}, \ldots, X_{t-1}, 0, \chi_{t+1}, \ldots, \chi_{T}\right) .
\end{aligned}
$$

Define the Malliavin difference $D_{t} Q$ by

$$
D_{t} Q:=Q^{t+}-Q^{t-} .
$$

Clearly, $Q$ is $\mathscr{F}_{k}$-measurable if and only if $D_{t} Q=0$ for all $t>k$, in which case $D_{k} Q$ is $\widetilde{F}_{k-1}$-measurable.

Lemma 2. (Martingale representation.) Let $L$ be a martingale difference process, i.e. an adapted process with $\mathbb{E}\left[L_{t+1} \mid \mathcal{F}_{t}\right]=0$. Then there exists a unique adapted process $Z$ such that

$$
L_{t+1}=Z_{t} M_{t+1} \quad \mathbb{P} \text {-almost surely for all } t \in \mathcal{T} \backslash\{T\} .
$$

Furthermore, $Z_{t}=D_{t+1} L_{t+1}$.

Proof. As in the martingale representation theorem for Markov chains in Elliott and Yang [21] and for general finite-state processes in Cohen and Elliott [9], [10], it is not difficult to see that $Z_{t-1}=D_{t} L_{t}$ is the unique solution to

$$
L_{t}^{t+}=Z_{t-1} M_{t}^{t+}=Z_{t-1}\left(1-p_{t-1}\right), \quad L_{t}^{t-}=Z_{t-1} M_{t}^{t-}=Z_{t-1}\left(-p_{t-1}\right) .
$$

Note that, unlike in Cohen and Elliott [10], as we are in the situation of a binomial model, this representation can be obtained with $M$ and $Z$ both scalar processes. The following two theorems are modifications of results in Cohen and Elliott [8] for this situation.

Theorem 1. (Existence of BSDE solutions.) Let $F: \Omega \times \mathcal{T} \times \mathbb{R} \times \mathbb{R} \rightarrow \mathbb{R}$ be an $\mathbb{F}$-adapted, integrable function (i.e. $F(\cdot, t, y, z) \in L^{1}\left(\mathcal{F}_{t}\right)$ for all $t$ ). Then the BSDE

$$
Y_{t+1}=Y_{t}-F\left(\omega, t, Y_{t}, Z_{t}\right)+Z_{t} M_{t+1}, \quad Y_{T}=Q
$$

has a unique solution $(Y, Z)$ for all $Q \in L^{1}\left(\mathcal{F}_{T}\right)$ if and only if the map

$$
y \mapsto y-F(\omega, t, y, z)
$$

is a bijection for all $(\omega, t, z) \in \Omega \times \mathcal{T} \times \mathbb{R}$.

Here, $(F, Q)$ are called the parameters of the BSDE, and will be called standard if Theorem 1 holds. Note that while the BSDE in Theorem 1 is in a differenced form, it can also be written in the form

$$
Y_{t}-\sum_{u=t}^{T-1} F\left(\omega, u, Y_{u}, Z_{u}\right)+\sum_{u=t}^{T-1} Z_{u} M_{u+1}=Q .
$$

Theorem 2. (Comparison theorem.) Consider the solutions $\left(Y^{1}, Z^{1}\right),\left(Y^{2}, Z^{2}\right)$ to two BSDEs with standard parameters $\left(F^{1}, Q^{1}\right),\left(F^{2}, Q^{2}\right)$, respectively. Suppose that for all $\omega, t, y, y^{\prime}, z$, and $z^{\prime}$,

(P1) $Q^{1} \geq Q^{2}$;

(P2) $F^{1}(\omega, t, y, z) \geq F^{2}(\omega, t, y, z)$; 
(P3) $F^{1}(\omega, t, y, z)-F^{1}\left(\omega, t, y, z^{\prime}\right) \geq \min \left\{\left(z-z^{\prime}\right) p_{t},\left(z-z^{\prime}\right)\left(p_{t}-1\right)\right\}$;

(P4) $y \mapsto y-F^{1}(\omega, t, y, z)$ is strictly increasing as a function of $y$.

Then $Y_{t}^{1} \geq Y_{t}^{2}$ for all $\omega, t$.

For a standard $F^{1}$, if properties (P3) and (P4) hold, then $F^{1}$ will be called balanced. Note that property (P3) also holds with the roles of $z$ and $z^{\prime}$ exchanged, which proves the following corollary.

Corollary 1. A balanced driver is Lipschitz in $z$ with Lipschitz constant at most 1.

Proof. Exchanging the role of $z$ and $z^{\prime}$, we have

$$
\left|F^{1}(\omega, t, y, z)-F^{1}\left(\omega, t, y, z^{\prime}\right)\right| \leq \max \left\{\left|\left(z-z^{\prime}\right) p_{t}\right|,\left|\left(z-z^{\prime}\right)\left(p_{t}-1\right)\right|\right\} \leq\left|z-z^{\prime}\right| .
$$

Conversely, the comparison theorem has the following implication for BSDEs with nonLipschitz drivers.

Theorem 3. Suppose $F$ is the driver of a BSDE and is not Lipschitz in $z$ with positive probability. Then there exist terminal values $Q^{1}, Q^{2}$ such that, for some t, the corresponding solutions satisfy $Y_{t+1}^{1} \leq Y_{t+1}^{2}$ almost surely, $Y_{t+1}^{1}<Y_{t+1}^{2}$ with positive probability, but $Y_{t}^{1}=Y_{t}^{2}$ almost surely.

Proof. As $F$ is not Lipschitz with positive probability, there exists some $t$, some set $A \in \mathcal{F}_{t}$, and some $y, z$, and $z^{\prime}$ such that

$$
F(\omega, t, y, z)-F\left(\omega, t, y, z^{\prime}\right)>\left|z-z^{\prime}\right|
$$

on $A$. Then, we define

$$
\begin{aligned}
Y_{t+1}^{1} & :=y-F(\cdot, t, y, z)+z M_{t+1}, \\
Y_{t+1}^{2} & := \begin{cases}y-F\left(\cdot, t, y, z^{\prime}\right)+z^{\prime} M_{t+1}, & \omega \in A, \\
Y_{t+1}^{1}, & \omega \in A^{c}\end{cases}
\end{aligned}
$$

so that on $A^{c}$ we have $Y_{t+1}^{1}=Y_{t+1}^{2}$ and on $A$,

$$
Y_{t+1}^{1}<y-F\left(\omega, t, y, z^{\prime}\right)-\left|z-z^{\prime}\right|+z M_{t+1}<y-F\left(\omega, t, y, z^{\prime}\right)+z^{\prime} M_{t+1}=Y_{t+1}^{2} .
$$

Hence, $Y_{t+1}^{1} \leq Y_{t+1}^{2}$, and the inequality is strict with positive probability. However, it is clear that the BSDE dynamics indicate that the corresponding solutions at time $t$ satisfy $Y_{t}^{1}=Y_{t}^{2}=y$. To complete this argument, we simply run the BSDE dynamics forward from time $t+1$ to time $T$, using an arbitrary process for $Z$, to generate the terminal values $Q^{1}$ and $Q^{2}$ with the desired solution.

\section{Dynamic convex risk measures and BSDEs}

In this section a BSDE for a dynamic convex risk measure is presented. Firstly, a conditional nonlinear expectation is defined, and the link between the conditional nonlinear expectation and the solution of a BSDE whose driver function and terminal condition satisfy certain properties is provided. Then the relationship between the conditional nonlinear expectation and the dynamic convex risk measure under certain mild conditions for the driver function is given. Finally, the driver function is explicitly identified.

Following Detlefsen and Scandolo [15], Bion-Nadal [4], and others, we state the following definition. 
Definition 2. A family of operators $\rho_{t}: L^{1}\left(\mathcal{F}_{T}\right) \rightarrow L^{1}\left(\mathcal{F}_{t}\right), t \in \mathcal{T}$ is called a dynamic convex risk measure if the following hold.

(i) (Monotonicity) For any $Q, V \in L^{1}\left(\mathcal{F}_{T}\right)$ with $Q(\omega) \geq V(\omega)$ for all $\omega \in \Omega$,

$$
\rho_{t}(Q) \leq \rho_{t}(V) \text { for all }(t, \omega) \in \mathcal{T} \times \Omega .
$$

(ii) (Dynamic constancy) For any $t \in \mathcal{T}$ and $Q \in L^{1}\left(\mathcal{F}_{t}\right)$,

$$
\rho_{t}(Q)=-Q \text {. }
$$

(iii) (Dynamic translability) For any $t \in \mathcal{T}, q \in L^{1}\left(\mathcal{F}_{t}\right)$, and $Q \in L^{1}\left(\mathcal{F}_{T}\right)$,

$$
\rho_{t}(Q+q)=\rho_{t}(Q)-q \text {. }
$$

(iv) (Dynamic convexity) For each $t \in \mathcal{T}, \rho_{t}$ is convex; that is, for any $Q_{1}, Q_{2} \in L^{1}\left(\mathcal{F}_{T}\right)$ and $\alpha \in L^{1}\left(\mathscr{F}_{t}\right)$ with $\alpha \in(0,1)$,

$$
\rho_{t}\left(\alpha Q_{1}+(1-\alpha) Q_{2}\right) \leq \alpha \rho_{t}\left(Q_{1}\right)+(1-\alpha) \rho_{t}\left(Q_{2}\right) .
$$

Note that $\rho_{0}$ is a (static) convex risk measure in the sense of Föllmer and Schied [23] and Frittelli and Rosazza-Gianin [25]. Also note that, from [15, Corollary 1], we know that $\rho$ is regular, that is, for each $t \in \mathcal{T}, A \in \mathcal{F}_{t}$, and $Q \in L^{1}\left(\mathcal{F}_{T}\right), \rho_{t}\left(\mathbf{1}_{A} Q\right)=\mathbf{1}_{A} \rho_{t}(Q)$, where $\mathbf{1}_{A}$ is the indicator function of a set $A$.

As we are working in a finite-state situation, the classical continuity of convex functions implies that any convex risk measure is continuous, i.e. if $X_{n} \rightarrow X$ pointwise then $\rho_{0}\left(X_{n}\right) \rightarrow$ $\rho(X)$. This implies (see[15, Theorem 1]) that there exists a family of probability measures $P_{\Lambda}$ on $(\Omega, \mathcal{F})$, and a random convex penalty function $\eta_{t}: P_{\Lambda} \rightarrow L^{1}\left(\mathcal{F}_{t}\right)$ such that

$$
\rho_{t}(Q)=\max _{\mathbb{P} \in P_{\Lambda}}\left\{-\mathbb{E}_{\mathbb{P}}\left[Q \mid \mathcal{F}_{t}\right]-\eta_{t}(\mathbb{P})\right\}
$$

and

$$
\min _{\mathbb{P} \in P_{\Lambda}}\left\{\eta_{t}(\mathbb{P})\right\}=0
$$

Furthermore, without loss of generality

$$
\eta_{t}(\mathbb{P})=\max _{Q \in L^{1}\left(\mathcal{F}_{T}\right)}\left\{-\mathbb{E}_{\mathbb{P}}\left[Q \mid \mathcal{F}_{t}\right]-\rho_{t}(Q)\right\},
$$

and so $\eta_{t}$ satisfies a regularity condition.

Lemma 3. Without loss of generality, for any probability measures $\mathcal{Q}, \mathbb{P} \in P_{\Lambda}$, we can 'paste' together the measures at time $t$ on a set $A \in \mathcal{F}_{t}$ to give a measure $\mathcal{R} \in P_{\Lambda}$ by

$$
\frac{\mathrm{d} \mathcal{R}}{\mathrm{d} \mathbb{P}}=\mathbf{1}_{A} \frac{\mathrm{d} \mathcal{Q} / \mathrm{d} \mathbb{P}}{\mathbb{E}\left[\mathrm{d} \mathcal{Q} / \mathrm{d} \mathbb{P} \mid \mathcal{F}_{t}\right]}+\mathbf{1}_{A^{c}},
$$

and $\eta_{t}$ satisfies $\eta_{t}(\mathcal{Q})=\eta_{t}(\mathcal{R})$.

Remark 1. In [15], the set $P_{\Lambda}$ is made to vary in time, and requires that $Q=\mathbb{P}$ on $\mathcal{F}_{t}$ for all $\mathcal{Q} \in P_{\Lambda}$. We do not make this restriction, however, and obtain this regularity property instead. The pasting of probability measures is fundamentally the same as the $m$-stability case considered in [13].

Note that, in this context, these are true maxima and minima taken pointwise (rather than essential suprema), due to the simplicity of the underlying binomial tree. 
Definition 3. A dynamic risk measure is said to be $\mathbb{F}$-consistent if it satisfies the recursivity property

$$
\rho_{s}\left(-\rho_{t}(Q)\right)=\rho_{s}(Q) \text { for all } Q \in L^{1}\left(\mathcal{F}_{T}\right), s \leq t .
$$

We now consider risk measures through the framework of BSDEs.

Theorem 4. The following two statements are equivalent:

(i) the family $\left\{\rho_{t} \mid t \in \mathcal{T}\right\}$ is an $\mathbb{F}$-consistent dynamic risk measure;

(ii) there exists a balanced driver $H$, which does not depend on $y$, is convex in $z$, and is such that for any $Q \in \mathcal{F}_{T}$,

$$
Y_{t}=\rho_{t}(Q),
$$

where $Y_{t}$ is the first component in the solution to a BSDE with parameters $(H,-Q)$.

Furthermore, the driver $H$ and the risk measure are explicitly related by

$$
H(\omega, t, z) \equiv \rho_{t}\left(-z M_{t+1}\right)
$$

Proof. It is clear that $\varepsilon_{t}(Q):=\rho_{t}(-Q)$ is a convex $\mathbb{F}$-consistent nonlinear expectation in the sense of Peng [30] (see also [32]). From [9, Theorem 5], we see that $\varepsilon_{t}(Q)$ has a representation as the solution to a BSDE with a balanced driver $H(\omega, t, z)=\varepsilon_{t}\left(z M_{t+1}\right)=\rho_{t}\left(-z M_{t+1}\right)$.

The following result is a direct consequence of Theorem 4 and Corollary 1. This is fundamentally different from the case of a continuous-time Wiener space, where it was shown in [11] that the driver relating to a dynamic convex risk measure is Lipschitz if and only if the risk measure is $\mathcal{E}^{\mu}$-dominated (we would like to thank the referee for pointing this out).

Corollary 2. In discrete time, the drivers of BSDEs corresponding to $\mathbb{F}$-consistent dynamic risk measures are Lipschitz.

Proof. This follows from Corollary 1, as the drivers are balanced.

Note that in the case of continuous-time BSDEs driven by Wiener processes, the characterization of the driver has already appeared in [30].

\section{BSDEs for prices and risks}

In this section we shall discuss the relationship between the BSDEs for the price and the risk of a contingent claim in the context of the binomial tree model. Consider a contingent claim maturing at time $T$ with payoff $G$ at time $T$, where $G$ is an $\mathcal{F}_{T}$-measurable random variable. We wish to understand the dynamics of the difference between the price of the claim $G$ and the risk of the discounted value of $G$, i.e. $\rho_{t}\left(G \cdot R^{-(T-t)}\right)$, where the dynamic convex risk measure $\left\{\rho_{t}\right\}$ is given by the solution to a BSDE and $R$ is the constant, one-period discount factor.

Let $\rho_{t}\left(G \cdot R^{-(T-t)}\right)=Y_{t}$ so that $Y_{t}$ is the risk of the claim $G$ evaluated at time $t$, where $(Y, Z)$ is the solution to the BSDE,

$$
G \cdot R^{-(T-t)}=Y_{t}-\sum_{u=t}^{T-1} H\left(\omega, u, Z_{u}\right)+\sum_{u=t}^{T-1} Z_{u} M_{u+1},
$$

which is taken under the real-world, or reference, measure $\mathbb{P}$. 
As in Cohen and Elliott [8], under the risk-neutral measure $\mathcal{Q}$ in the binomial tree model, we have the following BSDE:

$$
G \cdot R^{-(T-t)}=Y_{t}-\sum_{u=t}^{T-1} \tilde{H}\left(\omega, u, Z_{u}\right)+\sum_{u=t}^{T-1} Z_{u} \tilde{M}_{u+1},
$$

where $\tilde{H}(\omega, t, z)=H(\omega, t, y, z)+\left(q_{t}-p_{t}\right) z ;\left\{\tilde{M}_{t}\right\}$ is the martingale difference process generated by $\mathcal{Q}$.

Let $V_{t}$ be the price of the claim $G$ at time $t$. Taking an expectation under $\mathcal{Q}$, as $\mathbb{E}^{\mathcal{Q}}\left[G \cdot R^{-(T-t)}\right]=V_{t}$, we have

$$
\begin{aligned}
Y_{t}-V_{t} & =\mathbb{E}^{\mathcal{Q}}\left[\sum_{u=t}^{T-1} \tilde{H}\left(\omega, u, Z_{u}\right) \mid \mathcal{F}_{t}\right] \\
& =\mathbb{E}^{\mathcal{Q}}\left[\sum_{u=t}^{T-1} H\left(\omega, u, Z_{u}\right)+\left(q_{u}-p_{u}\right) Z_{u} \mid \mathcal{F}_{t}\right] \\
& =\mathbb{E}^{\mathcal{Q}}\left[\sum_{u=t}^{T-1} H\left(\omega, u, Z_{u}\right) \mid \mathcal{F}_{t}\right]-\mathbb{E}^{\mathcal{Q}}\left[\sum_{u=t}^{T-1}\left(p_{u}-q_{u}\right) Z_{u} \mid \mathcal{F}_{t}\right] \\
& =\mathbb{E}^{\mathcal{Q}}\left[Y_{t+1}-V_{t+1} \mid \mathcal{F}_{t}\right]+H\left(\omega, t, Z_{t}\right)-\left(p_{t}-q_{t}\right) Z_{t} .
\end{aligned}
$$

This decomposition seems quite surprising as it can be given the following interpretation. The term ' $\left(p_{u}-q_{u}\right) Z_{u}$ ' may be interpreted as the market risk-premium associated with the position $Z_{u}$. We have also shown that $H(\omega, t, z)=\rho_{t}\left(-z M_{t+1}\right)$, and so this quantity can be thought of as a personal risk-premium associated with the position $z$, given by the riskmeasure $\rho_{t}$.

Consequently, the difference between the risk and the price of the discounted contingent claim $G \cdot R^{-(T-t)}$ is decomposed into three components: the market price of their future difference, plus the personal evaluation of the risk associated with the one-step position $Z_{t}$, compensated by the market premium associated with that position.

\section{Mathematical properties of some specific risk measures}

In this section a particular type of dynamic convex risk measures introduced by stochastic distortion probabilities is first considered in the binomial tree framework and its time consistency is discussed. Then the conditions for the one-step law invariance of nonlinear expectation are identified. Finally, a dynamic entropic risk measure, which is another type of dynamic convex risk measures, is also discussed and its BSDE representation is given.

\subsection{Risk measures from stochastic distortions}

We now specify a form of dynamic convex risk measure by introducing the concept of 'stochastic distortion probabilities'. The notion of 'stochastic distortion probabilities' is related to those of distortion probabilities in a binomial tree model in [5] and stochastic intervals for subjective appreciation rates in [22].

Consider, for each $t \in \mathcal{T} \backslash\{T\}$, a stochastic interval of 'distortion factors', say $\Lambda_{t}:=$ $\left[\lambda_{t}^{L}, \lambda_{t}^{U}\right]$, where

(i) $\lambda_{t}^{L}$ and $\lambda_{t}^{U}$ are $\mathcal{F}_{t}$-measurable;

(ii) $0 \leq \lambda_{t}^{L} \leq \lambda_{t}^{U} \leq \infty, \mathbb{P}$-almost surely. 
Define an $F$-adapted process of 'stochastic distortion factors' $\lambda:=\left\{\lambda_{t} \mid t \in \mathcal{T} \backslash\{T\}\right\}$ on $(\Omega, \mathcal{F}, \mathbb{P})$ such that $\lambda_{t} \in \Lambda_{t}$. Write

$$
\Lambda:=\prod_{t=0}^{T-1} \Lambda_{t}
$$

For each $\lambda:=\left(\lambda_{0}, \lambda_{1}, \ldots, \lambda_{T-1}\right) \in \Lambda$, we take the stochastically distorted 'up probability' $p_{t}^{\lambda_{t}}$ in the place of the corresponding physical probability $p_{t}$. As $p_{t} \in[0,1]$, it follows that $p_{t}^{\lambda_{t}} \in[0,1]$. Consequently, for each $\lambda \in \Lambda$, we can use Lemma 1 to define the probability measure $\mathbb{P}_{\lambda}$ under which

$$
\mathbb{E}^{\lambda}\left[X_{t+1} \mid \mathscr{F}_{t}\right]=p_{t}^{\lambda_{t}} .
$$

Here $\mathbb{E}^{\lambda}$ is an expectation under $\mathbb{P}_{\lambda}$.

For each $\lambda \in \Lambda, \mathbb{P}_{\lambda}$ is absolutely continuous with respect to $\mathbb{P}$ on $\left(\Omega, \mathscr{F}_{T}\right)$. Furthermore, if $\lambda_{t}^{L}>0$ and $\lambda_{t}^{U}<\infty$ for all $t \in \mathcal{T} \backslash\{T\}, \mathbb{P}_{\lambda}$ is equivalent to $\mathbb{P}$ on $\mathcal{F}_{T}$. Then a family of equivalent probability measures $P_{\Lambda}:=\left\{\mathbb{P}^{\lambda} \mid \lambda \in \Lambda\right\}$ with index set $\Lambda$ is generated by 'stochastic distortion probabilities'.

Now, for a convex penalty function $\eta_{t}: P_{\Lambda} \rightarrow L^{1}\left(\mathcal{F}_{t}\right)$ satisfying the regularity property of Lemma 3,

$$
\rho_{t}(Q)=\sup _{\lambda \in \Lambda}\left\{-\mathbb{E}^{\lambda}\left[Q \mid \mathcal{F}_{t}\right]-\eta_{t}\left(\mathbb{P}_{\lambda}\right)\right\}
$$

The idea of 'stochastic distortion probabilities' generalizes the concept of 'constant distortion probabilities' used in actuarial science for premium calculations; see, e.g. [37] and [38]). In a recent paper by Cherny and Madan [7], the concept of 'constant distorted probabilities, or distributions' was used to develop a family of performance measures which are suitable for non-Gaussian return distributions. These constant distortions were then used by Eberlein and Madan [16] to measure hedge fund performance.

Theorem 5. If $\Lambda_{t}=[0, \infty]$ for all t then any probability measure on $(\Omega, \mathcal{F})$ can be generated by a stochastic distortion.

Proof. For any measure $\tilde{\mathbb{P}}$, let $\tilde{p}_{t}=\mathbb{E}^{\tilde{\mathbb{P}}}\left[\mathcal{X}_{t+1} \mid \mathcal{F}_{t}\right]$. Then define

$$
\lambda_{t}=\frac{\ln \left(p_{t}\right)}{\ln \left(\tilde{p}_{t}\right)} \in[0, \infty] \text {. }
$$

It is easy to see that the process $\lambda_{t}$ is the stochastic distortion relating $\mathbb{P}$ and $\tilde{\mathbb{P}}$.

Theorem 6. The risk measures generated by stochastic distortion probabilities are $\mathbb{F}$-consistent if and only if $\eta$ is of the form

$$
\eta_{t}\left(\mathbb{P}_{\lambda}\right)=f\left(\omega, t, \lambda_{t}\right)+\mathbb{E}^{\lambda}\left[\eta_{t+1}\left(\mathbb{P}_{\lambda}\right) \mid \mathcal{F}_{t}\right]=\mathbb{E}^{\lambda}\left[\sum_{u \geq t} f\left(\omega, u, \lambda_{u}\right) \mid \mathcal{F}_{t}\right]
$$

for some adapted function $f$.

Proof. We know that

$$
\rho_{t}(Q)=\sup _{\lambda \in \Lambda}\left\{-\mathbb{E}^{\lambda}\left[Q \mid \mathcal{F}_{t}\right]-\eta_{t}\left(\mathbb{P}_{\lambda}\right)\right\}
$$

and by the $\mathbb{F}$-consistency

$$
\rho_{t}(Q)=\rho_{t}\left(-\rho_{t+1}(Q)\right)=\sup _{\lambda \in \Lambda}\left\{\mathbb{E}^{\lambda}\left[\rho_{t+1}(Q) \mid \mathcal{F}_{t}\right]-\eta_{t}\left(\mathbb{P}_{\lambda}\right)\right\}
$$


Define

$$
f\left(\omega, t, \lambda_{t}\right):=\inf _{\left\{\bar{\lambda} \in \Lambda: \bar{\lambda}_{t}=\lambda_{t}\right\}} \eta_{t}\left(\mathbb{P}_{\bar{\lambda}}\right) .
$$

Then, as $\mathbb{E}^{\bar{\lambda}}\left[V \mid \mathcal{F}_{t}\right]=\mathbb{E}^{\lambda}\left[V \mid \mathcal{F}_{t}\right]$ for all $\bar{\lambda} \in \Lambda$ with $\bar{\lambda}_{t}=\lambda_{t}$, and all $V \in L^{1}\left(\mathcal{F}_{t+1}\right)$,

$$
\rho_{t}(Q)=\sup _{\lambda \in \Lambda}\left\{\mathbb{E}^{\lambda}\left[\rho_{t+1}(Q) \mid \mathcal{F}_{t}\right]-f\left(\omega, t, \lambda_{t}\right)\right\}
$$

We now write

$$
\rho_{t}(Q)=\sup _{\lambda \in \Lambda}\left\{\mathbb{E}^{\lambda}\left[\sup _{\lambda^{\prime} \in \Lambda}\left\{-\mathbb{E}^{\lambda^{\prime}}\left[Q \mid \mathcal{F}_{t+1}\right]-\eta_{t+1}\left(\mathbb{P}_{\lambda^{\prime}}\right)\right\} \mid \mathcal{F}_{t}\right]-f\left(\omega, t, \lambda_{t}\right)\right\} .
$$

Clearly, this implies

$$
\begin{aligned}
\rho_{t}(Q) & \geq \sup _{\lambda \in \Lambda}\left\{\mathbb{E}^{\lambda}\left[-\mathbb{E}^{\lambda}\left[Q \mid \mathcal{F}_{t+1}\right]-\eta_{t+1}\left(\mathbb{P}_{\lambda}\right) \mid \mathcal{F}_{t}\right]-f\left(\omega, t, \lambda_{t}\right)\right\} \\
& =\sup _{\lambda \in \Lambda}\left\{\mathbb{E}^{\lambda}\left[-Q \mid \mathcal{F}_{t}\right]-\left[\mathbb{E}^{\lambda}\left[\eta_{t+1}\left(\mathbb{P}_{\lambda}\right) \mid \mathcal{F}_{t}\right]+f\left(\omega, t, \lambda_{t}\right)\right]\right\} .
\end{aligned}
$$

From the definition of the supremum, for every $\varepsilon>0$ there exists a $\lambda^{\prime} \in \Lambda$ such that

$$
\rho_{t}(Q) \leq \sup _{\lambda \in \Lambda}\left\{\mathbb{E}^{\lambda}\left[-\mathbb{E}^{\lambda^{\prime}}\left[Q \mid \mathcal{F}_{t+1}\right]-\eta_{t+1}\left(\mathbb{P}_{\lambda^{\prime}}\right) \mid \mathcal{F}_{t}\right]-f\left(\omega, t, \lambda_{t}\right)\right\}+\varepsilon
$$

For a given $\lambda^{\prime}$, let $\left(\lambda \lambda^{\prime}\right) \in \Lambda$ be the process defined by

$$
\left(\lambda \lambda^{\prime}\right)_{s}= \begin{cases}\lambda_{s}, & s \leq t, \\ \lambda_{s}^{\prime}, & s>t .\end{cases}
$$

By the regularity property of $\eta$, we have $\eta_{t+1}\left(\mathbb{P}_{\left(\lambda \lambda^{\prime}\right)}\right)=\eta_{t+1}\left(\mathbb{P}_{\lambda^{\prime}}\right)$. Hence,

$$
\rho_{t}(Q) \leq \sup _{\lambda \in \Lambda}\left\{\mathbb{E}^{\left(\lambda \lambda^{\prime}\right)}\left[-Q-\eta_{t+1}\left(\mathbb{P}_{\left(\lambda \lambda^{\prime}\right)}\right) \mid \mathcal{F}_{t}\right]-f\left(\omega, t, \lambda_{t}\right)\right\}+\varepsilon
$$

and, as $\left(\lambda \lambda^{\prime}\right) \in \Lambda$, we have

$$
\rho_{t}(Q) \leq \sup _{\lambda \in \Lambda}\left\{\mathbb{E}^{\lambda}\left[-Q \mid \mathcal{F}_{t}\right]-\left[\mathbb{E}^{\lambda}\left[\eta_{t+1}\left(\mathbb{P}_{\lambda}\right) \mid \mathcal{F}_{t}\right]+f\left(\omega, t, \lambda_{t}\right)\right]\right\}+\varepsilon .
$$

As $\varepsilon$ is arbitrary, combined with (2), we have

$$
\begin{aligned}
\rho_{t}(Q) & =\sup _{\lambda \in \Lambda}\left\{\mathbb{E}^{\lambda}\left[-Q \mid \mathcal{F}_{t}\right]-\left[\mathbb{E}^{\lambda}\left[\eta_{t+1}\left(\mathbb{P}_{\lambda}\right) \mid \mathcal{F}_{t}\right]+f\left(\omega, t, \lambda_{t}\right)\right]\right\} \\
& =\sup _{\lambda \in \Lambda}\left\{-\mathbb{E}^{\lambda}\left[Q \mid \mathcal{F}_{t}\right]-\eta_{t}\left(\mathbb{P}_{\lambda}\right)\right\},
\end{aligned}
$$

and so $\eta_{t}\left(\mathbb{P}_{\lambda}\right)=\mathbb{E}^{\lambda}\left[\eta_{t+1}\left(\mathbb{P}_{\lambda}\right) \mid \mathcal{F}_{t}\right]+f\left(\omega, t, \lambda_{t}\right)$ as desired. The second equality follows clearly by recursion.

Remark 2. This result can be seen as analogous to the cocycle condition obtained by BionNadal [4] in a general context. Furthermore, the representation of the penalty function in Theorem 6 can be seen as a discrete-time version of the representation of the penalty function in a continuous-time Brownian setting by Delbaen et al. [14], where the latter is obtained by replacing the summation in the former by an integral. 
Theorem 7. The risk measures generated by the stochastic distortion probabilities are $\mathbb{F}$-consistent if and only if $\rho_{t}(Q)$ is given by a BSDE with parameters $(H,-Q)$, where

$$
\left.H(\omega, t, z)=\sup _{\lambda \in \Lambda}\left\{z\left(p_{t}^{\lambda_{t}}-p_{t}\right)-f\left(\omega, t, \lambda_{t}\right)\right)\right\}
$$

Here, $f$ is defined as in Theorem 6.

Proof. First note that as $z M_{t+1} \in L^{1}\left(\mathcal{F}_{t+1}\right)$, from (1) we have

$$
\rho_{t}\left(-z M_{t+1}\right)=\sup _{\lambda \in \Lambda}\left\{\mathbb{E}^{\lambda}\left[z M_{t+1} \mid \mathcal{F}_{t}\right]-f\left(\omega, t, \lambda_{t}\right)\right\} .
$$

Then, from Theorem 4,

$$
\begin{aligned}
H(\omega, t, z) & =\rho_{t}\left(-z M_{t+1}\right) \\
& =\sup _{\lambda \in \Lambda}\left\{\mathbb{E}^{\lambda}\left[z M_{t+1} \mid \mathcal{F}_{t}\right]-f\left(\omega, t, \lambda_{t}\right)\right\} \\
& =\sup _{\lambda \in \Lambda}\left\{z \mathbb{E}^{\lambda}\left[M_{t+1} \mid \mathcal{F}_{t}\right]-f\left(\omega, t, \lambda_{t}\right)\right\} \\
& =\sup _{\lambda \in \Lambda}\left\{z\left(p_{t}^{\lambda_{t}}-p_{t}\right)-f\left(\omega, t, \lambda_{t}\right)\right\} .
\end{aligned}
$$

Again, by Theorem 4,

$$
H(\omega, t, z)=\varepsilon_{t}\left(z M_{t+1}\right)
$$

and the result follows.

\subsection{One-step law invariance}

We now seek to understand under what conditions our nonlinear expectation satisfies a degree of law invariance. Recent work of Kupper and Schachermayer [29] has shown that the only nonlinear expectation which is time-consistent, relevant, and law-invariant with respect to the terminal condition is the exponential utility, i.e. the negative of the entropic risk (see below). However, we consider here under what properties law invariance is satisfied with respect to the values in the next step. This may be interpreted as local law invariance. This weaker condition allows far greater freedom in the choice of risk measures, while maintaining some of the intuition behind law-invariant risk measures.

Definition 4. A risk measure $\rho$ is said to be one-step law-invariant if, for any $t$, for any two $\mathcal{F}_{t+1}$ measurable random variables $X, X^{\prime}$ with equal conditional laws $F_{X \mid \mathcal{F}_{t}}=F_{X^{\prime}} \mid \mathcal{F}_{t}$, we have $\rho_{t}(X)=\rho_{t}\left(X^{\prime}\right)$.

Theorem 8. A risk measure is one-step law-invariant if and only if $\rho_{t}\left(z M_{t+1}\right)$ is an even function of $z$ whenever $p_{t}=\frac{1}{2}$.

Proof. When $p_{t}=\frac{1}{2}$, if $X$ and $X^{\prime}$ have the same law then it is easy to show that $X-\mathbb{E}\left[X \mid \mathcal{F}_{t}\right]= \pm\left[X^{\prime}-\mathbb{E}\left[X^{\prime} \mid \mathcal{F}_{t}\right]\right]$. Hence, in the BSDE representation, the solution processes $Z$ satisfy $Z^{X}= \pm Z^{X^{\prime}}$. Hence, the risk measures are the same if and only if $H\left(t, Z^{X}\right)=H\left(t, Z^{X^{\prime}}\right)$, i.e. $H$ is an even function of $z$. As $H(\omega, t, z)=\rho\left(-z M_{t+1}\right)$, the result is proven.

Note that whenever $p_{t} \neq \frac{1}{2}$, two $\mathcal{F}_{t+1}$ random variables can agree in law if and only if they are almost surely equal. Hence, one-step law-invariance is trivial. The result of Theorem 8 may 
suggest that in continuous time there may be a form of 'short-term' law invariance for classical BSDEs driven by a Brownian motion whenever the driver is an even function. As our focus is on the simple binomial model, we shall not pursue this further here.

In the following theorem, we strengthen the results in Theorem 8 by considering equivalent probability measures.

Theorem 9. A risk measure is one-step law-invariant for every equivalent probability measure if and only if

$$
\rho_{t}\left(z\left(\mathcal{X}_{t+1}-\frac{1}{2}\right)\right)
$$

is an even function of $z$ for all $\omega, t$.

Proof. Using the preceding theorem, the BSDE representation, and a change of measures, $\rho_{t}$ is one-step law-invariant if and only if

$$
\rho_{t}\left(z M_{t+1}\right)-\left(\frac{1}{2}-p_{t}\right) z=\rho\left(z\left(\mathcal{X}_{t+1}-\frac{1}{2}\right)\right)
$$

is an even function of $z$, where the equality is due to translation invariance and the definition of $M_{t+1}$.

This theorem seems quite surprising, as law invariance is typically closely tied to the choice of probability laws.

\subsection{Entropic risk}

In this section, we consider a particular case of dynamic convex risk measure, namely, a dynamic entropic risk measure, which is related to the certainty equivalence under an exponential utility function in a dynamic setting.

Definition 5. A dynamic entropic risk measure $\left\{\rho_{t}^{\mathbb{E}}(\cdot) \mid t \in \mathcal{T}\right\}$ of the position $Q$ is defined by

$$
\rho_{t}^{\mathbb{E}}(Q):=\frac{1}{\gamma} \ln \left\{\mathbb{E}\left[\exp (-\gamma Q) \mid \mathcal{F}_{t}\right]\right\}, \quad t \in \mathcal{T},
$$

where $\gamma$ is the risk aversion parameter.

We now show that the dynamic entropic risk measure is obtained from a stochastic distortion when the penalty function is given by a multiple of the conditional relative entropy between $\mathbb{P}^{\lambda}$ and $\mathbb{P}$ given $\mathcal{F}_{t}$.

Theorem 10. Suppose that, for each $t \in \mathcal{T}$,

$$
\rho_{t}(Q):=\frac{1}{\gamma} \ln \left\{\mathbb{E}\left[\exp (-\gamma Q) \mid \mathcal{F}_{t}\right]\right\} .
$$

Then $\rho_{t}(Q)$ can be represented as

$$
\rho_{t}(Q)=\sup _{\lambda \in \Lambda}\left\{\mathbb{E}^{\lambda}\left[-Q \mid \mathcal{F}_{t}\right]-\eta_{t}\left(\mathbb{P}_{\lambda}\right)\right\}
$$

where $\Lambda=(0, \infty)^{T}$ and the penalty function $\eta_{t}\left(\mathbb{P}_{\lambda}\right)$ is given by

$$
\eta_{t}\left(\mathbb{P}_{\lambda}\right):=\frac{1}{\gamma} \mathbb{E}\left[\left(\frac{\mathrm{d} \mathbb{P}^{\lambda}}{\mathrm{d} \mathbb{P}}\right) \ln \left(\frac{\mathrm{d} \mathbb{P}^{\lambda}}{\mathrm{d} \mathbb{P}}\right) \mid \mathcal{F}_{t}\right] .
$$


Proof. As $\Lambda_{t}=(0, \infty)$ for all $t$, we know from Theorem 5 that any probability measure can be written as a distortion measure. The result is then standard; see, e.g. [3] or [15].

The following theorem gives the BSDE governing the evolution of $\left\{\rho_{t}^{\mathbb{E}} \mid t \in \mathcal{T}\right\}$ over time.

Theorem 11. The dynamic entropic risk measure $\left\{\rho_{t}^{\mathbb{E}} \mid t \in \mathcal{T}\right\}$ satisfies the following BSDE:

$$
\rho_{t}^{\mathbb{E}}-Z_{t}^{\mathbb{E}} p_{t}-\frac{1}{\gamma} \ln \left[1-p_{t}\left(1-\mathrm{e}^{-\gamma Z_{t}^{\mathbb{E}}}\right)\right]+Z_{t}^{\mathbb{E}} M_{t+1}=\rho_{t+1}^{\mathbb{E}}, \quad \rho_{T}^{\mathbb{E}}=-Q .
$$

Proof. We know that $\rho^{\mathbb{E}}$ comes from a BSDE with driver

$$
H^{\mathbb{E}}(\omega, t, z)=\rho_{t}\left(-z M_{t+1}\right)
$$

and so

$$
\begin{aligned}
H^{\mathbb{E}}(\omega, t, z) & =-\frac{1}{\gamma} \ln \mathbb{E}\left[\exp \left(\gamma z M_{t+1}\right) \mid \mathcal{F}_{t}\right] \\
& =-\frac{1}{\gamma} \ln \left[\left(p_{t} \mathrm{e}^{\gamma z\left(1-p_{t}\right)}+\left(1-p_{t}\right) \mathrm{e}^{-\gamma z p_{t}}\right)\right] \\
& =-\frac{1}{\gamma} \ln \left[\mathrm{e}^{-\gamma z p_{t}}\left(1-p_{t}\left(1-\mathrm{e}^{\gamma z}\right)\right)\right] \\
& =z p_{t}-\frac{1}{\gamma} \ln \left[1-p_{t}\left(1-\mathrm{e}^{\gamma z}\right)\right] .
\end{aligned}
$$

For the special case where $p_{t}=\frac{1}{2}$, this further reduces to

$$
\rho_{t}\left(-z M_{t+1}\right)=H^{\mathbb{E}}(\omega, t, z)=-\frac{1}{\gamma} \ln \left[\cosh \left(\frac{\gamma z}{2}\right)\right],
$$

which is clearly an even function of $z$ as required by Theorem 8 .

Remark 3. It is worth recalling at this point that the driver we observe for the entropic risk measure $\rho^{\mathbb{E}}$ must be Lipschitz continuous as it must be balanced (cf. Corollary 1). This stands in contrast to the continuous-time case, where the exponential utility is known to be given by a BSDE with quadratic driver $-\gamma z^{2} / 2$.

Nevertheless, by expanding $H^{\mathbb{E}}(\omega, t, z)$ in $z$ using Taylor's expansion, we have

$$
\begin{aligned}
H^{\mathbb{E}}(\omega, t, z) & =-z p_{t}-\frac{1}{\gamma} \ln \left[1-p_{t}\left(1-\mathrm{e}^{-\gamma z}\right)\right] \\
& =-\gamma p_{t}\left(1-p_{t}\right) \frac{z^{2}}{2}+o\left(z^{3}\right) \\
& \approx-\gamma p_{t}\left(1-p_{t}\right) \frac{z^{2}}{2} .
\end{aligned}
$$

This further connects the discrete-time theory with the continuous-time one for Brownian motion, Note that the additional term $p_{t}\left(1-p_{t}\right)$ is the correction due to the conditional variance of $M_{t+1}$. Consequently, $\left\{\rho_{t}^{\mathbb{E}} \mid t \in \mathcal{T}\right\}$ satisfies approximately the following BSDE:

$$
\rho_{t}^{\mathbb{E}}+\frac{\gamma p_{t}\left(1-p_{t}\right)}{2}\left(Z_{t}^{\mathbb{E}}\right)^{2}+Z_{t}^{\mathbb{E}} M_{t+1}=\rho_{t+1}^{\mathbb{E}}
$$

Note that the solution of this BSDE may not define a dynamic entropic risk measure since its driver is not exactly equal to $\rho_{t}\left(-z M_{t+1}\right)$. The solution may only serve as an approximation to a dynamic entropic risk measure at best. 
Remark 4. The relative entropy penalty function can be written as

$$
\eta_{t}\left(\mathbb{P}_{\lambda}\right):=\frac{1}{\gamma} \mathbb{E}\left[\left(\frac{\mathrm{d} \mathbb{P}^{\lambda}}{\mathrm{d} \mathbb{P}}\right) \ln \left(\frac{\mathrm{d} \mathbb{P}^{\lambda}}{\mathrm{d} \mathbb{P}}\right) \mid \mathcal{F}_{t}\right]=\mathbb{E}^{\lambda}\left[\sum_{u \geq t} f\left(\omega, u, \lambda_{u}\right) \mid \mathcal{F}_{t}\right]
$$

where $f\left(\omega, t, \lambda_{u}\right)=\ln \left(p_{u}^{\lambda_{u}-1}\right)=\left(\lambda_{u}-1\right) \ln \left(p_{u}\right)$. This is of the form required by Theorem 6 .

\section{Acknowledgements}

The authors would like to thank the anonymous referee for helpful comments. Robert J. Elliott and Tak Kuen Siu would like to acknowledge the Discovery Grants from the Australian Research Council (ARC) (project Nos. DP1096243 and DP130103517). Samuel Cohen thanks the Oxford-Man Institute for Quantitative Finance for support.

\section{References}

[1] Artzner, P., Delbaen, F., Eber, J.-M. and Heath, D. (1999). Coherent measures of risk. Math. Finance 9, 203-228.

[2] ArtZner, P. et al. (2002). Coherent multiperiod risk measurement. Preprint, Department of Mathematics, ETH, Zürich.

[3] Barrieu, P. AND El Karoui, N. (2004). Optimal derivatives design under dynamic risk measures. In Mathematics of Finance (Contemp. Math. 351), American Mathematical Society, Providence, RI, pp. 13-25.

[4] BION-NADAL, J. (2004). Conditional risk measure and robust representation of convex conditional risk measures. CMAP Preprint 557, École Polytechnique, Paris.

[5] Boyle, P., SiU, T. K. ANd Yang, H. (2002). Risk and probability measures. Risk 15, 53-57.

[6] Cheridito, P. And Stadje, M. (2013). BS $\Delta$ Es and BSDEs with non-Lipschitz drivers: comparison, convergence and robustness. Bernoulli 19, 1047-1085.

[7] Cherny, A. and Madan, D. (2009). New measures of performance evaluation. Rev. Financ. Stud. 22, 2571-2606.

[8] Cohen, S. N. And Elliott, R. J. (2009). A general theory of backward stochastic difference equations. Presentation Notes. University of Adelaide and University of Calgary.

[9] Cohen, S. N. And Elliott, R. J. (2010). A general theory of finite state backward stochastic difference equations. Stoch. Process. Appl. 120, 442-466.

[10] Cohen, S. N. and Elliott, R. J. (2011). Backward stochastic difference equations with finite states. In Stochastic Analysis with Financial Applications (Progr. Prob. 65), Birkhäuser, Basel, pp. 33-42.

[11] Coquet, F., Hu, Y., Mémin, J. And Peng, S. (2002). Filtration-consistent nonlinear expectations and related g-expectations. Prob. Theory Relat. Fields 123, 1-27.

[12] Cvitanić, J. and Karatzas, I. (1999). On dynamic measures of risk. Finance Stoch. 3, 451-482.

[13] Delbaen, F. (2006). The structure of $m$-stable sets and in particular of the set of risk neutral measures. In Memoriam Paul-André Meyer (Lecture Notes Math. 1874), Springer, Berlin, pp. 215-258.

[14] Delbaen, F., Peng, S. and Rosazza-Gianin, E. (2010). Representation of the penalty term of dynamic concave utilities. Finance Stoch. 14, 449-472.

[15] Detlefsen, K. and Scandolo, G. (2005). Conditional and dynamic convex risk measures. Finance Stoch. 9, 539-561.

[16] Eberlein, E. And Madan, D. B. (2009). Hedge fund performance: sources and measures. Internat. J. Theoret. Appl. Finance 12, 267-282.

[17] El Karoui, N., Peng, S. and Quenez, M. C. (1997). Backward stochastic differential equations in finance. Math. Finance 7, 1-71.

[18] Elliott, R. J. AND Kopp, P. E. (2005). Mathematics of Financial Markets, 2nd edn. Springer, New York.

[19] Elliott, R. J. AND SiU, T. K. (2012). A BSDE approach to convex risk measures for derivative securities. Stoch. Anal. Appl. 30, 1083-1101.

[20] Elliott, R. J. AND SiU, T. K. (2013). Reflected backward stochastic differential equations, convex risk measures and American options. Stoch. Anal. Appl. 31, 1077-1096.

[21] Elliott, R. J. and Yang, H. (1994). How to count and guess well: discrete adaptive filters. Appl. Math. Optimization 30, 51-78.

[22] Elliott, R. J., SiU, T. K. AND Chan, L. (2008). A PDE approach for risk measures for derivatives with regime switching. Ann. Finance 4, 55-74. 
[23] Föllmer, H. ANd Schied, A. (2002). Convex measures of risk and trading constraints. Finance Stoch. 6, 429-447.

[24] Föllmer, H. AND Schied, A. (2004). Stochastic Finance: An Introduction in Discrete Time (De Gruyter Studies Math. 27), 2nd edn. De Gruyter, Berlin.

[25] Frittelli, M. and Rosazza-Gianin, E. (2002). Putting order in risk measures. J. Banking Finance 26, 1473-1486.

[26] Frittelli, M. and Rosazza-Gianin, E. (2004). Dynamic convex risk measures. In Risk Measures for the 21st Century, ed. G. Szegö, John Wiley, Chichester, pp. 227-248.

[27] Jobert, A. And Rogers, L. C. G. (2008). Valuations and dynamic convex risk measures. Math. Finance 18, $1-22$.

[28] Klöppel, S. AND Schweizer, M. (2007). Dynamic indifference valuation via convex risk measures. Math. Finance 17, 599-627.

[29] Kupper, M. and Schachermayer, W. (2009). Representation results for law invariant time consistent functions. Math. Financial Econom. 2, 189-210.

[30] PENG, S. (1997). Backward SDE and related g-expectation. In Backward Stochastic Differential Equations (Pitman Res. Notes Math. Ser. 364), Longman, Harlow, pp. 141-159.

[31] Privault, N. (2009). Stochastic Analysis in Discrete and Continuous Settings with Normal Martingales. Springer, Berlin.

[32] Rosazza-Gianin, E. (2006). Risk measures via g-expectations. Insurance Math. Econom. 39, $19-34$.

[33] SIU, T. K. (2012). Functional Itô's calculus and dynamic convex risk measures for derivative securities. Commun. Stoch. Anal. 6, 339-358.

[34] SIU, T. K. AND YANG, H. (1999). Subjective risk measures: Bayesian predictive scenarios analysis. Insurance Math. Econom. 25, 157-169.

[35] SiU, T. K. And YAng, H. (2000). A PDE approach to risk measures of derivatives. Appl. Math. Finance 7, 211-228.

[36] SiU, T. K., Tong, H. AND YANG, H. (2001). Bayesian risk measures for derivatives via random Esscher transform. N. Amer. Actuarial J. 5, 78-91.

[37] WANG, S. S. (2000). A class of distortion operators for pricing financial and insurance risks. J. Risk Insurance 67, 15-36.

[38] Wang, S. S. AND Young, V. R. (1998). Risk-adjusted credibility premiums using distorted probabilities. Scand. Actuarial J. 1998, 143-165.

[39] Wang, T. (1999). A class of dynamic risk measures. Preprint. Faculty of Commerce and Business Administration, University of British Columbia. 